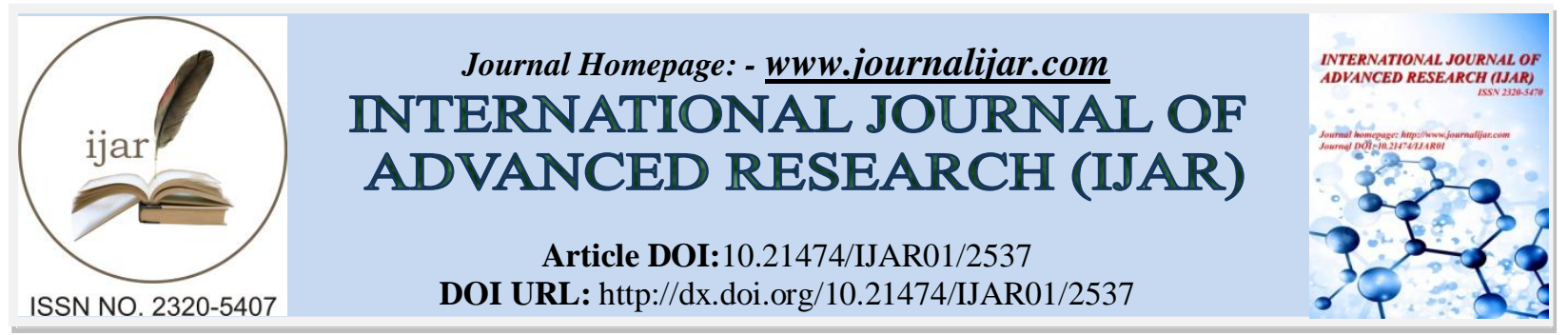

RESEARCH ARTICLE

\title{
DESIGN A METHOD TO HANDLE THE RISKY POST-DISCHARGE ISSUES IN KING ABDULAZIZ UNIVERSITY HOSPITAL.
}
Wejdan A. Al-Ghamdi, Jawaher S. Al-Otaibi, Kholoud A. Hassna, Shoaa K. Al-Dhahri, Rawan K. Althobaiti, Rogaya M. Alshugair, Ohoud B. Almehwari, Arwa M. Aljehani, Eman A. Alsahafi, Marwa A. Aljehani, Aishah S. Alasmari, Amal S. Al-Malki, Maryam M. Al-Shayook and Hadeel A. Katib

\section{Manuscript Info}

…………………

Manuscript History

Received: 25 October 2016

Final Accepted: 23 November 2016

Published: December 2016

\section{Abstract}

The post-discharge period considered to be critical because the transition from hospital to home exposes patients to stressful events. Using phone call after patient discharge as a tool to identify discharging gaps will help to develop an effective discharge plan and improve patient outcomes.

Key words:-

Post-discharge, Patient safety,

Phone system, Follow-up, Hospital

quality,

Medical issues,

Health education.

Copy Right, IJAR, 2016,. All rights reserved.

\section{Background:-}

The post-discharge period considered to be critical because the transition from hospital to home exposes patients to stressful events. During this time, patients may face many problems such as inadequate communication between them and the health care providers, lack of follow-up \& discontinuity of care which will affect patient management (1).

An appropriate and effective discharge plan can ensure patients' quality and continuity of care at home. In order to develop an effective discharge plan, it's necessary to identify the problems that face patients after leaving the hospital, but most of these problems are not detectable until patients readmitted again as a result of the defects in the transitional care (2).

Post-discharge phone calls enabling professional-patient interaction to ensure patient understanding delivery of information and monitor patient condition in an interactive practical method. Using phone call after patient discharge as a tool to identify discharging gaps will help to develop an effective discharge plan (2).

\section{Methodology:-}

\section{Design, Setting, and Patients:-}

The research is an observational study. It introduced to the medical department at King Abdulaziz University Hospital in Jeddah, Saudi Arabia. The study was approved by the research ethics committee of the faculty of medicine, King Abdul-Aziz University.

Consecutive patients admitted to this service between October 10th 2015 till November 23rd, 2015 were screened for eligibility,those who discharged home from the: female medical ward, male medical ward, coronary care unit 
(CCU) and isolation unit (main inclusion criteria) besides who have contact information and cooperated. We excluded the following: long-standing patients and don't meet the inclusion criteria.

152 patients enrolled in the study, 102 patients were called using a structured questionnaire (checklist) to assess their post-discharge status.

\section{Data collection:-}

\section{Step 1:-}

The study was done by 16 members of 6th-year medical students, they were tracking discharges from the medicine service daily and ensuring the patients understood the purpose of the study and a verbal consent was taken before discharge. The phone call has done during 48-72 $\mathrm{h}$ after leaving the hospital and extended 7-10 minutes for each patient. The caller made up 4 attempts during the $72 \mathrm{~h}$ using all available contact information numbers.

Post-discharge phone call (PDPC) checklist was formulated in reference to Project RED (Re-Engineered Discharge) Training Program and Project BOOST® Implementation Toolkit and reviewed by the supervisor of the study.

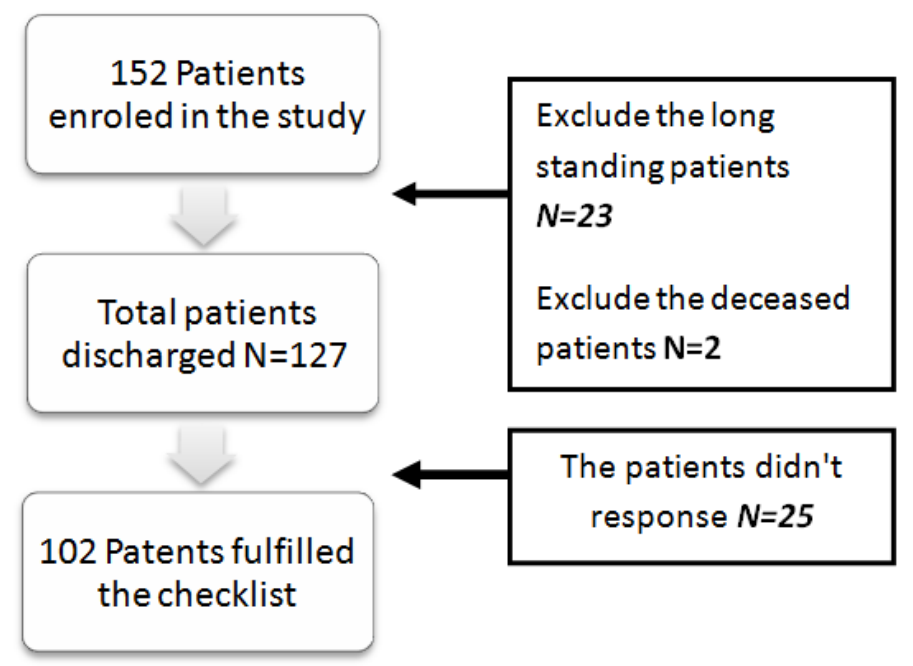

Figure :- Shows the number of patientswho enrolled in the study.

The checklist made of two parts. The first one is about patient information; it filed through interviewing the patient before leaving the hospital and by reviewing his/her discharging summary using open-ended questions.

The second part was divided into four categories: health status diagnosis, patient medications, clarification of appointments and emergency state in series of questions that require yes, no or nonapplicable choices .Table 1 shows the categories in the checklist and the number of questions.

Table 1:- Categories of questions in the checklist.

\begin{tabular}{|c|c|}
\hline The Category: & No of Questions: \\
\hline Health Status Diagnosis & 6 \\
\hline Patients Medication & 5 \\
\hline Clarification of Appointment & 6 \\
\hline Emergency State & 1 \\
\hline
\end{tabular}




\section{Step 2:-}

The patient feedback was done between 1st-5th December 2015. Patients who completed the checklist of step 1 successfully had been selected randomly to get their satisfaction about the service by another phone call. The randomization performed by ranking the patients from 1 to 102 and the phone call has been done to a patient with even numbers.In case the patient doesn't answer, the caller moves to another patient above or below the chosen number. 50 calls were conducted. The questionnaire asked if the (PDPC) checklist covers the important points in the discharge status, rating the service, opinion of implementingthis service in other hospital's departments and any suggestions to improve the quality of the service.

The responses of both step 1 and step 2 were documented in the electronic form using a designed template and an Excel spreadsheet.

Data Analysis: The data were analyzed using SPSS 20.0 with descriptive frequencies and chi-square.

\section{Results:-}

During the study period, 152 of patients participated, 102 patients responded to our calls, 50 patients were not enrolled into the study because 23 of them were long standing, 25 not responds to our calls and 2 were deceased. According to the admission rate $46.1 \%$ of participants were their first admission while $53.9 \%$ was not. The majority of patients were students in high school or below 59.9\%, 24.51\% and $20.59 \%$ were in Bachelor's degree and above and non-educated respectively. Issues identified in the calls were grouped in table 2.

Table 2:- The post-discharge checklist answers:

\begin{tabular}{|l|c|}
\hline Identified issue: & Response \\
\hline Medical team didn't explain diagnosis to the patients & $16.7 \%$ \\
\hline $\begin{array}{l}\text { Patients who didn't know the measures / precautions about their disease as: BP, FBG, body weight, } \\
\text { etc. }\end{array}$ & $40.5 \%$ \\
\hline Medical team didn't explain to the patients the way of using their medications & $5.1 \%$ \\
\hline Patients have questions about their medications & $27.5 \%$ \\
\hline Patients didn't have all the prescribed medications & $15.3 \%$ \\
\hline Patients can't offer the medications in their prescription & $38.8 \%$ \\
\hline $\begin{array}{l}\text { Patients didn't know that they have to continue using their medications for the decided period, even } \\
\text { after they run out of them }\end{array}$ & $13.2 \%$ \\
\hline Patients got lost reaching their clinic & $33.3 \%$ \\
\hline Patients didn't know what to do in case they miss their appointment & $48.4 \%$ \\
\hline The medical team didn'taware the patients about the important symptoms that need emergency visit & $51 \%$ \\
\hline
\end{tabular}

For the health status diagnosis related questions and patients' medication parts, most of the patients who have been contacted admit that they received enough information about their diagnosis and its management which are accepted. We look to achieve better outcomes. The other category that considers patient appointment shows some extent of the defects and almost half of the patients don't know what the conditions they have to seek medical orientation for.These need to be improved because both will affect the patients' outcomes and their compliance for the follow-up. 
We also found that the numbers of patients who were approached by hospital social workers present in figure 2 .

Figure 2: Shows patients results of hospital social workers visiting before the discharge

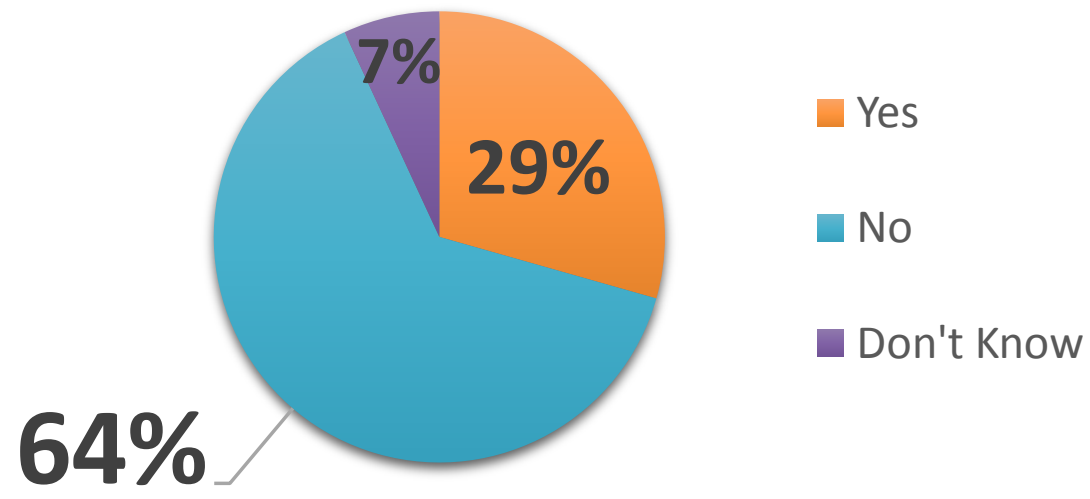

Then, this service is necessary to be encouraged more in the multidisciplinary healthcare team because it provides multi useful utilities to support patients and their families (educationally, socially, financially etc.).

Analyzing patient feedback regarding the (PDPC) gave us a direct insight into what's working well and what's not. Out of 50 calls were made, 46 of them fulfilled the questionnaire and 4 didn't answer. $91.3 \%$ of the patients said that the checklist questions cover the important points in their discharging status. All of the given answers highly recommend applying this service in all hospital departments. Patients' evaluations were: $58.7 \%$ as "very good" and $41.3 \%$ as a good process in improving after hospitalization status.

\section{Discussion:-}

The initial feedback we obtained from the patients shows significant results regarding the main four aspects of the checklist. If we use these results properly, we can address a well discharge plan and prevent major post-discharge adverts events.

Lacking of previous local trials was the major conflict to begin this study. The study was done by 6th-year medical students starting from designing the checklist till making the phone calls. Our aim was to assess the presence of discharging errors so it didn't involve any kind of interventions. A group of patients demonstrates differences in age, educational levels, clinical status, comorbidities and number of admissions. We used phone call in order to popularize a method among all patients regardless their discrepancies. The checklist was written in Arabic and translated into English. We believe that our checklist covers the main aspects of discharging process. We didn't face any complaints or inquiries out of it. The checklist also considers the patient's hospital course and we tried to correlate this information with health status as reported by them or their careers after discharge. In the satisfaction survey, patients praised post-discharge phone call program.

Based on research done in Toronto hospital (3) and according to hospital policy which provides follow up appointment one week after discharge, we contacted patients in the first 48-72 hours after discharge. Others (4) made the phone call 2 weeks after hospitalization. We didn't involve the age in our exclusion criteria, unlike other studies which gave age an important factor, (2), (4). We refer to patient discharge report as a base for patient condition post discharge. This study didn't include carers in the questionnaire (4) because the primary aim of it was to investigate the possibility of discharge issues. The answers obtained through our checklist were strict, directed 
with closed end questions and not time consuming. We took into account patients' knowledge of personal measures/ precautions that related to their disease, their medication and follow up compliance, (4), (5). We didn't consider the readmission rate in our study. This may be in part because a proportion of readmissions are not preventable despite the excellent care coordination (5). Although preventing readmission is important, there are other outcomes which are also important to patients and their health, including disease control, medication adherence and symptom management (4). Referring to (1), patients prefer an interactive voice response system more than the follow up calls.

The mentioned results can't be generalized on all KAUH outcomes because the study applied to the medical department only and we don't have a vision if the checklist can be applied to all departments in KAUH and/or other medical centers.

There were some limitations regarding sample; we found significant long-standing patients $15.1 \%$, about $16.4 \%$ didn't answer when we contacted them and non Arabic/English speakers weren't easy to communicate with.

The final data suggest that this quality project can be favorably addressed by well-designed patient care system involving medical team, Information Technology department, patient relation and social workers in the immediate post-discharge period.

\section{Conclusion:-}

Our study supports the value of centering a post-discharge phone call program in the health care practice. The success of our project is based on the ability of the IT to enlist the checklist in the hospitals' software system. The patient relation should: inform the patients and take the consent, get their accessible phone number. For the medical teams, they should provide a clear discharge note for each patient includes: diagnosis, health status at discharge procedure has been done in the hospital, prescribed medications, date of the next appointment / clinic, last investigations ordered. For making the phone calls, either: 1- well trained medical staff (nurses, interns, etc.), 2quality improving organization or 3- hospitals' patient relation service.

We would like to apply this quality study in all hospital departments with modifiable checklist that address their individuality. We also recommend doing some sort of interventions through the phone call as needed to evaluate its possibility to reduce readmission rate. Long following up the study outcomes is necessary to guide the health care system toward the main issues that need to be emphasized to the patients through the discharging process.

\section{Acknowledgements:-}

We would like to thank:

1. Dr.Kamal Al-Ghalayini Assistant Professor, Chairman of Medical Department at KAUH \& the Study Supervisor.

2. Dr. MahaS.Aljadani and Dr. Noor A.Alamoudi 6th year medical students for their works during the project and data analysis.

3. Dr.Abeer Arab Anesthesiologist \& Clinical Simulation Specialist at KAUH, Coordinator of Patient Safety module for the 6th-year medical students at medicine collage-KAU.

4. Dr. Amro Alhibshi Chairman of King Abdulaziz University Hospital for supporting the project.

\section{References:-}

1. FORSTER, A. J., BOYLE, L., SHOJANIA, K. G., FEASBY, T. E. \& VAN WALRAVEN, C. 2009. Identifying patients with post-discharge care problems using an interactive voice response system. J Gen Intern Med, 24, 520-5.

2. BULL M.J., ROBERTS J. 2001. Component of a proper hospital discharge for elders Journal of Advanced Nursing, $35,571-581$.

3. DOREEN OUELLET, J. F. 2010. Post Discharge phone Call Program - Improving Transitions in Care. A guidebook to patient safety leading practices: 2010;advancing patient safety through ideas and innovations. Toronto, Ontario,Canda: Ontario hospital association.

4. DRISCOLL A., 2000. Managing post-discharge care at home: an analysis of patients' and their careers' perceptions of information received during their stay in the hospital. Journal of Advanced Nursing, 31, 1165-1173.

5. TANG, N., FUJIMOTO, J. \& KARLINER, L. 2014. Evaluation of a primary care-based post-discharge phone call program: keeping the primary care practice at the center of post-hospitalization care transition. J Gen Intern Med, 29, 1513-8. 